\title{
Mechanical stresses produced by a light beam
}

\author{
Radi I. Khrapko* \\ Moscow Aviation Institute, Moscow, Russia \\ (Received 17 June 2007; final version received 9 September 2007)

\begin{abstract}
A circularly polarized electromagnetic beam is considered, which is absorbed by a plane, and the mechanical stress produced in the plane by the beam is calculated. It is shown that the central part of the beam produces a torque at the central region of the plane due to the spin of the beam, and the wall of the beam produces an additional torque due to orbital angular momentum of the beam. The total torque acting on the plane equals twice the power of the beam divided by the frequency. This fact contradicts the standard electrodynamics, which predicts the torque equals power of the beam divided by frequency, and means the electrodynamics, as well as the whole classical field theory, is incomplete. Introducing the spin tensor corrects the electrodynamics.
\end{abstract}

Keywords: electrodynamics torque; angular momentum; spin tensor

\section{Does the electrodynamics' spin tensor exist?}

As is well known, photons carry spin, energy, momentum and angular momentum that is a moment of the momentum relative to a given point or to a given axis. Energy and momentum of electromagnetic waves are described by the Maxwell energy-momentum tensor (density)

$$
T^{\lambda \mu}=-g^{\lambda \alpha} F_{\alpha \nu} F^{\mu \nu}+g^{\lambda \mu} F_{\alpha \beta} F^{\alpha \beta} / 4
$$

where $F^{\mu \nu}=-F^{\nu \mu}, F_{\mu \nu}=F^{\alpha \beta} g_{\mu \alpha} g_{\nu \beta}(\mu, \nu, \ldots=0,1,2,3)$ is the field strength tensor. For example, $P^{i}=\int_{V} T^{i 0} \mathrm{~d} V$ is the momentum of a wave inside a volume $V$, and $\mathrm{d} W=\int_{a} T^{0 i} \mathrm{~d} a_{i} \mathrm{~d} t$ is the energy that has flowed through the area $a$ in the time $\mathrm{d} t$. The angular momentum, that is a moment of the momentum, can be defined as [1]

$$
L^{i j}=\int_{V} 2 x^{[i} T^{j] 0} \mathrm{~d} V=\int_{V} \boldsymbol{r} \times(\boldsymbol{E} \times \boldsymbol{B}) \mathrm{d} V
$$

and this construction must be named an orbital angular momentum. However, modern electrodynamics has no description of spin.

\footnotetext{
*Email: khrapko_ri@hotmail.com
} 
The electrodynamics starts from the canonical Lagrangian [2, (4-111)], $L_{c}=-F_{\mu \nu} F^{\mu \nu} / 4$. Then, by the Lagrange formalism, the canonical energy-momentum tensor [2, (4-113)]

$$
T_{c}^{\lambda \mu}=\partial^{\lambda} A_{\alpha} \frac{\partial L_{c}}{\partial\left(\partial_{\mu} A_{\alpha}\right)}-g^{\lambda \mu} L_{c}=-\partial^{\lambda} A_{\alpha} F^{\mu \alpha}+g^{\lambda \mu} F_{\alpha \beta} F^{\alpha \beta} / 4
$$

and the canonical total angular momentum tensor [2, (4-147)]

$$
J_{c}^{\lambda \mu \nu}=2 x^{[\lambda} T_{c}^{\mu] \nu}+Y_{c}^{\lambda \mu \nu}
$$

are obtained. Here

$$
\Upsilon_{c}^{\lambda \mu \nu}=-2 A^{[\lambda} \delta_{\alpha}^{\mu]} \frac{\partial L_{c}}{\partial\left(\partial_{\nu} A_{\alpha}\right)}=-2 A^{[\lambda} F^{\mu] \nu}
$$

is the canonical spin tensor [2, (4-150)]. Its space component is $\boldsymbol{E} \times \boldsymbol{A}$ :

$$
Y_{c}^{i j 0}=\boldsymbol{E} \times \boldsymbol{A} .
$$

The sense of a spin tensor $Y^{\lambda \mu \nu}$ is as follows. The component $Y^{i j 0}$ is a volume density of spin. This means that $\mathrm{d} S^{i j}=\Upsilon^{i j 0} \mathrm{~d} V$ is the spin of the electromagnetic field inside the spatial element $\mathrm{d} V$. The component $Y^{i j k}$ is a flux density of spin flowing in the direction of the $x^{k}$ axis. For example, $\mathrm{d} S_{z} / \mathrm{d} t=\mathrm{d} S^{x y} / \mathrm{d} t=\mathrm{d} \tau^{x y}=Y^{x y z} \mathrm{~d} a_{z}$ is the $z$-component of the spin flux passing through the surface element $\mathrm{d} a_{z}$ per unit time, i.e. the torque acting on the element.

The sense of a total angular momentum tensor means that the total angular momentum of an element $\mathrm{d} V_{\nu}$ is $\mathrm{d} J^{\lambda \mu}=J^{\lambda \mu \nu} \mathrm{d} V_{v}=2 x^{[\lambda} T^{\mu] \nu} \mathrm{d} V_{\nu}+Y^{\lambda \mu \nu} \mathrm{d} V_{\nu}$. The corresponding integral is

$$
J^{\lambda \mu}=L^{\lambda \mu}+S^{\lambda \mu}=\int_{V} 2 x^{[\lambda} T^{\mu] \nu} \mathrm{d} V_{\nu}+\int_{V} \Upsilon^{\lambda \mu \nu} \mathrm{d} V_{\nu}
$$

It consists of two terms: the first term involves a moment of momentum and represents an orbital angular momentum; the second term is spin. It must be emphasized that a moment of momentum cannot represent spin. This idea is discussed in the paper [3], which was written in response to [4].

However, the canonical tensors (3), (4), (5) are not electrodynamics tensors. They obviously contradict experiments. For example, consider a circularly polarized plane wave,

$$
\begin{gathered}
E^{x}=\cos (z-t), \quad E^{y}=-\sin (z-t), \quad B^{x}=\sin (z-t), \quad B^{y}=\cos (z-t), \\
A^{x}=\sin (z-t), \quad A^{y}=\cos (z-t)
\end{gathered}
$$

(for short we set $k=\omega=1$ ). A calculation of components of the canonical spin tensor (5) yields

$$
\begin{aligned}
Y_{c}^{x y 0} & =1, \quad Y_{c}^{x y z}=1, \\
Y_{c}^{z x y} & =A^{x} B_{x}=\sin ^{2}(z-t), \\
Y_{c}^{y z x} & =A^{y} B_{y}=\cos ^{2}(z-t) .
\end{aligned}
$$


This result is absurd, because, though $Y_{c}^{x y 0}$ and $Y_{c}^{x y z}$ are adequate, the result means that there are spin fluxes in the directions which are transverse to the direction of the wave propagation.

To modify the invalid canonical tensors, specific terms, $-\partial_{\nu} \tilde{\Upsilon}_{c}^{\lambda \mu \nu} / 2$ and $-\partial_{\kappa}\left(x\left[\lambda \tilde{\Upsilon}_{c}^{\mu] \nu \kappa}\right)\right.$, are added to the tensors (3) and (4) [5,6] (here $\tilde{Y}_{c}^{\lambda \mu \nu} \stackrel{\text { def }}{=} \Upsilon_{c}^{\lambda \mu \nu}-Y_{c}^{\mu \nu \lambda}+Y_{c}^{\nu \lambda \mu}=-2 A^{\lambda^{c}} F^{\mu \nu}$ ). This procedure gives the standard energy-momentum tensor $T_{s t}^{\lambda \mu}$ and the standard total angular momentum tensor $J_{s t}^{\lambda \mu \nu}$,

$$
\begin{aligned}
& T_{s t}^{\lambda \mu}=T_{c}^{\lambda \mu}-\partial_{\nu} \tilde{\Upsilon}_{c}^{\lambda \mu \nu} / 2=-\partial^{\lambda} A_{\nu} F^{\mu \nu}+g^{\lambda \mu} F_{\alpha \beta} F^{\alpha \beta} / 4+\partial_{\nu}\left(A^{\lambda} F^{\mu \nu}\right), \\
& J_{s t}^{\lambda \mu \nu}=J_{c}^{\lambda \mu \nu}-\partial_{\kappa}\left(x^{[\lambda} \tilde{Y}_{c}^{\mu] \nu \kappa}\right) .
\end{aligned}
$$

Unfortunately, the energy-momentum tensor $T_{s t}^{\lambda \mu}(8)$ is obviously invalid, as well as the canonical energy-momentum tensor (3). So, the (Belinfante-Rosenfeld) procedure $[5,6]$ is unsuccessful, and the tensors (8), (9) are never used. But to make matters worse the procedure gives the standard spin tensor which equals zero! That is, the procedure eliminates classical spin at all:

$$
Y_{s t}^{\lambda \mu \nu}=J_{s t}^{\lambda \mu \nu}-2 x^{[\lambda} T_{s t}^{\mu] \nu}=Y_{c}^{\lambda \mu \nu}+2 A^{[\lambda} F^{\mu] \nu}=0 .
$$

That is why a spin term is absent in Equation (11). Nevertheless, physicists understand they cannot shut their eyes to the existence of classical electrodynamics spin. And they proclaim spin is in the moment of the momentum (2). That is, the moment of momentum represents the total angular momentum, orbital angular momentum plus spin. In other words, Equation (2) encompasses both the spin and orbital angular momentum density of a light beam $[2,7-10]$ :

$$
J^{i j}=L^{i j}+S^{i j}=\int_{V} 2 x^{[i} T^{j] 0} \mathrm{~d} V=\int_{V} \boldsymbol{r} \times(\boldsymbol{E} \times \boldsymbol{B}) \mathrm{d} V .
$$

Contrary to this paradigm, we introduce a spin tensor $\Upsilon^{\lambda \mu \nu}$ into the modern electrodynamics [11-16], i.e. we complete the electrodynamics by introducing the spin tensor, i.e. we claim the total angular momentum consists of the moment of momentum (2) and a spin term, i.e. we claim Equation (11) is wrong, i.e. we state the moment of momentum does not contain spin at all:

$$
J^{i j}=L^{i j}+S^{i j}=\int_{V}\left(2 x^{[i} T^{j] 0}+Y^{i j 0}\right) \mathrm{d} V=\int_{V} \boldsymbol{r} \times(\boldsymbol{E} \times \boldsymbol{B}) \mathrm{d} V+\int_{V} \Upsilon^{i j 0} \mathrm{~d} V .
$$

In contrast to the procedure [5,6], we offered other addends to the canonical energymomentum and spin tensors, viz. $\partial_{\nu} A^{\lambda} F^{\mu \nu}$ and $2 A^{[\lambda} \partial^{\mu]} A^{v}$, which satisfy the equations $\partial_{\nu} A^{[\lambda} F^{\mu] \nu}=\partial_{\nu}\left(A^{[\lambda} \partial^{\mu]} A^{v}\right), \partial_{\nu} A^{v}=0$. As a result, we arrive at the Maxwell tensor (1) $T^{\lambda \mu}$ instead of (8) and, at long last, at our spin tensor $Y^{\lambda \mu \nu}$ (15) instead the zero:

$$
\begin{aligned}
T_{c}^{\lambda \mu}+\partial_{\nu} A^{\lambda} F^{\mu \nu} & =T^{\lambda \mu}, \\
Y_{c}^{\lambda \mu \nu}+2 A^{[\lambda} \partial^{\mu]} A^{v} & =2 A^{[\lambda} \partial^{|v|} A^{\mu]}, \\
Y^{\lambda \mu \nu} & =\left(A^{[\lambda} \partial^{|\nu|} A^{\mu]}+\Pi^{[\lambda} \partial^{|\nu|} \Pi^{\mu]}\right) ;
\end{aligned}
$$


here $A^{\lambda}$ and $\Pi^{\lambda}$ are magnetic and electric vector potentials which satisfy $2 \partial_{[\mu} A_{\nu]}=F_{\mu \nu}$, $2 \partial_{[\mu} \Pi_{\nu]}=-e_{\mu \nu \alpha \beta} F^{\alpha \beta}$. A relation between $\Pi$ and $F$ can be readily obtained in the vector form as follows. If $\operatorname{div} \boldsymbol{E}=0$, then $\boldsymbol{E}=\operatorname{curl} \Pi$. And if $\partial \boldsymbol{E} / \partial t=\operatorname{curl} \boldsymbol{H}$, then $\partial \Pi / \partial t=\boldsymbol{H}$. This reasoning is analogous to the common: if $\operatorname{div} \boldsymbol{B}=0$, then $\boldsymbol{B}=\operatorname{curl} \boldsymbol{A}$. And if $\partial \boldsymbol{B} / \partial t=-\operatorname{curl} \boldsymbol{E}$, then $\partial \boldsymbol{A} / \partial t=-\boldsymbol{E}$.

The difference between our statement (12) and the common Equation (11) is verifiable. The cardinal question is, what angular momentum flux, i.e. torque $\tau$, does a circularly polarized light beam of power $P$ without an azimuth phase structure carry? The common answer, according to (11), is

$$
\tau=\mathrm{d} J / \mathrm{d} t=P / \omega
$$

our answer, according to (12), is

$$
\tau=\mathrm{d} J / \mathrm{d} t=2 P / \omega
$$

Statements (12) and (17) are also valid in the case of plane waves or a beam which is much larger than the particle under action if $P$ is the power absorbed by the particle.

Some theoretical calculations, in particular, the calculation of absorption of a circularly polarized light beam in a dielectric $[9,14]$, the calculation of a radiation of spin by a rotating electric dipole [16], as well as numerous experimental works [10,17-20], confirm our result (17) as is shown in [15]. At the same time results of $[21,22]$ confirm the common formula (16).

Another manifestation of the spin tensor concerns the mechanical stress that arises in a target absorbing a circularly polarized electromagnetic beam. A stress tensor density $T_{\wedge}^{i j}$ describes this stress. The quantity $T_{\wedge}^{i j}$ is calculated in Sections 2 and 3 of the present paper for the cases (11) and (12), respectively. The stress in the case (11) is proven to be in contradiction with the evidence. The two terms of (12) describe two different torques which equal each other but are exerted in different places. This fact, in our opinion, excludes double-counting of a torque.

In Section 4, it is shown that the so-called decomposition (11) of the moment of the Poynting vector in an orbital and spin angular momentums [7,23-26] is false.

\section{Absorbing of the moment of the Poynting vector flux}

According to (11), a plane wave traveling in the $z$-direction and with infinite extension in the $x y$-directions can have no angular momentum about the $z$-axis because $\boldsymbol{E} \times \boldsymbol{B}$ is in the $z$-direction and $[\boldsymbol{r} \times(\boldsymbol{E} \times \boldsymbol{B})]_{z}=0$. However, this is no longer the case for a wave with finite extension in the $x y$-plane. Consider a circularly polarized beam with its axis in the $z$-direction and traveling in this direction [7]

$$
\boldsymbol{E}=\exp (\mathrm{i} z-\mathrm{i} t)\left[\boldsymbol{x}+\mathrm{i} \boldsymbol{y}+\boldsymbol{z}\left(\mathrm{i} \partial_{x}-\partial_{y}\right)\right] E_{0}(x, y), \quad \boldsymbol{B}=-\mathrm{i} \boldsymbol{E} .
$$

Here $E_{0}(x, y)=E_{0}(r)$ is the electric field of the beam. For short we set $\omega=k=c=1$. Let $E_{0}(r)$ be explicitly made constant over a large central region of the beam and confine the variation of the function from this constant value to zero in order to lie within a wall of small thickness $\delta$, which lies a distance $r=R_{0}$ from the axis. It can be shown that the wall 


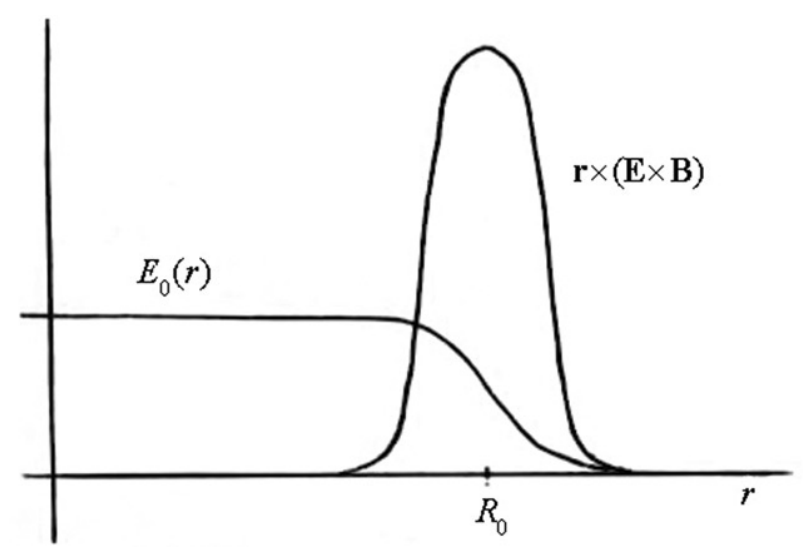

Figure 1. The profile of the electric field of the beam and the angular momentum density.

of such a beam gives a finite contribution to $J_{z}$ because the $\boldsymbol{E}$ and $\boldsymbol{B}$ fields have components parallel to the wave vector (the field lines are closed loops) and the energy flow has a component perpendicular to the wave vector. Please see our Figure 1, or Figure 1 from [23], or Figure 9.3 from [24]. The circulating energy flow in the beam implies the existence of angular momentum, whose direction is along the direction of propagation. Since the fields are identically zero outside the wall and constant inside the wall region, the wall region is the only one in which the $z$-component of angular momentum does not vanish.

The profile $E_{0}(r)$ of the beam may be Gaussian [27],

$$
\begin{aligned}
& E_{0}=\frac{(2 / \pi)^{1 / 2}}{w} \exp \left\{-\frac{r^{2}}{w^{2}}\left(1-\mathrm{i} \frac{z}{z_{R}}\right)+\mathrm{i} \arctan \frac{z}{z_{R}}\right\}, \\
& r^{2}=x^{2}+y^{2}, \quad w^{2}=\frac{2\left(z^{2}+z_{R}^{2}\right)}{z_{R}},
\end{aligned}
$$

but it doesn't matter. We set

$$
\int_{-\infty}^{+\infty} E_{0}^{2} \mathrm{~d} x \mathrm{~d} y=\int_{0}^{\infty} E_{0}^{2} 2 \pi r \mathrm{~d} r=1
$$

so, the power of the beam is

$$
P=\left\langle\int(\boldsymbol{E} \times \boldsymbol{B})_{z} \mathrm{~d} x \mathrm{~d} y\right\rangle=\int \Re\left(E_{x} \bar{B}_{y}-E_{y} \bar{B}_{x}\right) \mathrm{d} x \mathrm{~d} y / 2=\int E_{0}^{2} \mathrm{~d} x \mathrm{~d} y=1
$$

(the over lines mark complex conjugate complex numbers).

We need also the $p^{x}, p^{y}$ components of momentum density:

$$
p^{x}=\left\langle(\boldsymbol{E} \times \boldsymbol{B})_{x}\right\rangle=\Re\left(E_{y} \bar{B}_{z}-E_{z} \bar{B}_{y}\right) / 2=\partial_{y} E_{0}^{2} / 2, \quad p^{y}=-\partial_{x} E_{0}^{2} / 2 .
$$


Let a plane $z=0$ absorb the beam. Then the plane, according to (16), must experience the torque

$$
\tau=P=1
$$

(we ignore the light pressure). We will get the stress tensor density $T_{\wedge}^{i k}$ of the plane.

We use the cylindrical coordinates $r, \phi, z$

$$
x=r \cos \phi, \quad y=r \sin \phi,
$$

with the metric

$$
\begin{aligned}
& \mathrm{d} l^{2}=\mathrm{d} r^{2}+r^{2} \mathrm{~d} \phi^{2}+\mathrm{d} z^{2}, \quad g_{r r}=1, \quad g_{\phi \phi}=r^{2}, \\
& g_{z z}=1, \quad\left(g_{\wedge}\right)^{1 / 2}=r, \quad g^{\phi \phi}=1 / r^{2} .
\end{aligned}
$$

The square root of the determinant of the metric tensor is a scalar density of weight +1 . Gothic symbols are usually applied to denote tensor densities [28]. We shall, instead, mark the density with the symbol 'wedge' at the level of bottom indices for a density of weight +1 and at the level of top indices for a density of weight -1 . A volume element and a surface element are densities of weight $-1, \mathrm{~d} V^{\wedge}=\mathrm{d} r \mathrm{~d} \phi \mathrm{d} z, \mathrm{~d} a^{\wedge}=\mathrm{d} r \mathrm{~d} \phi$ as well as the absolute antisymmetric density $e_{i j k}^{\wedge}$, which equals \pm 1 , or 0 .

$\phi$-component $p^{\phi}$ is obtained by the formulae $p^{i}=p^{a} \partial_{a}^{i}, \partial_{a}=\partial_{a}^{i} \partial_{i}$, i.e.

$$
p^{\phi}=p^{x} \partial_{x}^{\phi}+p^{y} \partial_{y}^{\phi}, \quad \partial_{x}=\partial_{x}^{r} \partial_{r}, \quad \partial_{y}=\partial_{y}^{r} \partial_{r},
$$

where the matrix elements $\partial_{a}^{i}$ are

$$
\partial_{x}^{\phi}=-y / r^{2}, \quad \partial_{y}^{\phi}=x / r^{2}, \quad \partial_{x}^{r}=x / r, \quad \partial_{y}^{r}=y / r .
$$

The physical component of momentum density $p^{\hat{\phi}}$ is equal to the coordinate component of the tensor density $p_{\wedge}^{\phi}$ :

$$
p_{\wedge}^{\phi}=p^{\phi}\left(g_{\wedge}\right)^{1 / 2}=p^{\hat{\phi}}\left(g^{\phi \phi}\right)^{1 / 2}\left(g_{\wedge}\right)^{1 / 2}=p^{\hat{\phi}}=-\partial_{r} E_{0}^{2} / 2 .
$$

So, because $c=1$, the tensor density $p_{\wedge}^{\phi}$ equals the momentum flux density, i.e. the force tensor density $f_{\wedge}^{\phi}$ :

$$
f_{\wedge}^{\phi}=-\partial_{r} E_{0}^{2} / 2
$$

This force density $f_{\wedge}^{i}$ acts on the absorbing plane $z=0$, thus

$$
\nabla_{k} T_{\wedge}^{i k}=f_{\wedge}^{i},
$$

where $\nabla_{k} T_{\wedge}^{i k}$ is the covariant divergence of the stress tensor density of the plane. As is known [28], $\nabla_{k} T_{\wedge}^{i k}=\partial_{k} T_{\wedge}^{i k}+\Gamma_{j k}^{i} T_{\wedge}^{j k}$, where $\Gamma_{j k}^{i}$ are the Christoffel symbols. The nonzero symbols are

$$
\Gamma_{\phi \phi}^{r}=-r, \quad \Gamma_{\phi r}^{\phi}=\Gamma_{r \phi}^{\phi}=1 / r, \quad \Gamma_{k r}^{k}=\Gamma_{r k}^{k}=1 / r
$$




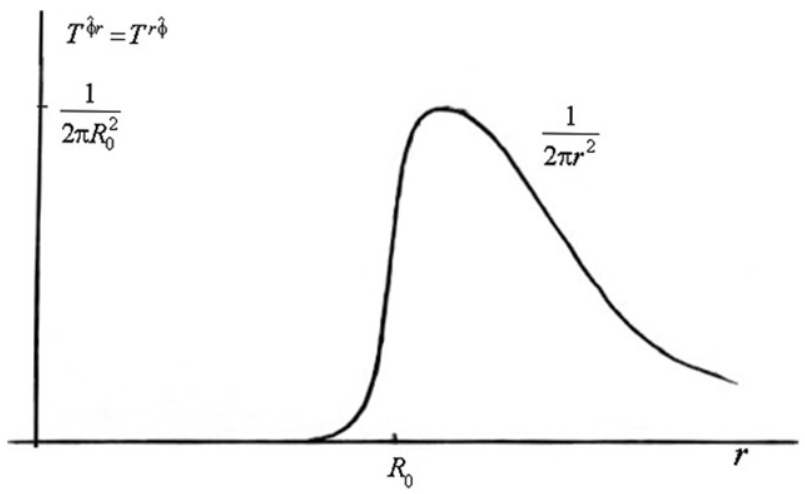

Figure 2. The component of the stress tensor arisen from the orbital angular momentum.

Because $T_{\wedge}^{\phi r}=T_{\wedge}^{r \phi}$, Equation (30) gives,

$$
\partial_{r} T_{\wedge}^{\phi r}+\Gamma_{j k}^{\phi} T_{\wedge}^{j k} \equiv \partial_{r} T_{\wedge}^{\phi r}+\Gamma_{\phi r}^{\phi} T_{\wedge}^{\phi r}+\Gamma_{r \phi}^{\phi} T_{\wedge}^{r \phi} \equiv \partial_{r} T_{\wedge}^{\phi r}+2 T_{\wedge}^{\phi r} / r=f_{\wedge}^{\phi} .
$$

This equation has a solution

$$
T_{\wedge}^{\phi r}=T^{\hat{\phi} r}=C(r) / r^{2}, \quad \partial_{r} C / r^{2}=-\partial_{r} E_{0}^{2} / 2 .
$$

It is easy to verify that $T_{\wedge}^{r r}=T_{\wedge}^{\phi \phi}=0$.

Integrating the equation for $C(r)$ yields

$$
C(r)=-\int_{0}^{r} r^{2} \mathrm{~d} E_{0}^{2} / 2=-r^{2} E_{0}^{2} / 2+\int_{0}^{r} r E_{0}^{2} \mathrm{~d} r .
$$

According to (34), $C=0$ and $T_{\wedge}^{\phi r}=0$ in the central region, where $E_{0}(r)=E_{0}(0)$. Thus, there is no mechanical stress in the central region of the target, according to the standard paradigm (4). This is depicted in Figure 2.

$C(r)$ increases up to $C_{\max }=\int_{0}^{\infty} r E_{0}^{2} \mathrm{~d} r=1 / 2 \pi$ in the wall region, in accordance with (20). Correspondingly,

$$
T_{\wedge}^{\phi r}=T^{\hat{\phi} r}=1 / 2 \pi r^{2}, \quad r>R_{0}
$$

outside the wall region. It is easy to verify that tensor density (35) satisfies $\nabla_{k} T_{\wedge}^{i k}=0$. A plot of $T^{\hat{\phi} r}(r)$ is given in Figure 2.

By the use of Equation (35), one can get the forces inside the target plane. An element $\mathrm{d} l$ of a circumference of radius $r$ experiences the force $\mathrm{d} F^{\hat{\phi}}=T^{\hat{\phi} r} \mathrm{~d} l=C(r) \mathrm{d} l / r^{2}$ and the torque $\mathrm{d} \tau=C(r) \mathrm{d} l / r$. Thus, the outside part of the plane experiences the torque

$$
\tau_{\text {orbit }}=\int_{l=0}^{l=2 \pi r} \frac{C(r)}{r} \mathrm{~d} l=2 \pi C(r),
$$


which increases up to the whole torque

$$
\tau_{\text {orbit }}=1, \quad \text { for } \quad r>R_{0}
$$

in accordance with (16) and (23).

However, a circularly polarized beam, as well as a circularly polarized plane wave, beyond doubt, acts on a central region of an absorbing plane by a torque. This torque arises from the fact that the dielectric constant $\varepsilon$ is a tensor. Consequently the electric intensity $\boldsymbol{E}$ is, in general, not parallel to the electric polarization $\boldsymbol{P}$ in the medium of the plane. The torque per unit volume produced by the action of the electric field on the polarization of the medium is [21]

$$
\tau / V=\boldsymbol{P} \times \boldsymbol{E} .
$$

But this torque is not connected with the moment of the Poynting vector (2) and (11).

Feynman repeated this explanation [29]. We quote [29] with some abridgements.

If we have a beam of light containing a large number of photons all circularly polarized the same way, it will carry angular momentum. Now remember what right circularly polarized light is, classically. It's described by an electric field so that the electric vector $\boldsymbol{E}$ goes in a circle - as drawn in Figure 17-5(a). Suppose that such a light shines on a plane which is going to absorb it - or at least some of it - and consider an atom in the plane according to the classical physics. We'll suppose that the atom is isotropic, so the result is that the electron moves in a circle, as shown in Figure 17-5(b). The electron is displaced at some displacement $\boldsymbol{r}$ from its equilibrium position at the origin and goes around with some phase lag with respect to the vector $\boldsymbol{E}$. The relation between $\boldsymbol{E}$ and $\boldsymbol{r}$ might be as shown in Figure 17-5(b). As time goes on, the electric field rotates and the displacement rotates with the same frequency, so their relative orientation stays the same. But look, there is angular momentum being poured into this electron, because there is always a torque about the origin. The torque is $e E_{t} r$ which must be equal to the rate of change of angular momentum $\mathrm{d} J_{z} / \mathrm{d} t$ :

$$
\mathrm{d} J_{z} / \mathrm{d} t=e E_{t} r
$$

This torque is not connected with the moment of the Poynting vector (2) and (11). The tangential component of the Poynting vector density is zero at the central region of the plane. Thus, the standard paradigm cannot explain this central region torque.

However, Allen and Padgett [30] try to explain the action of a circularly polarized plane wave by a torque on a central region of an absorbing plane in the frame of the standard paradigm. They cut the wave into coaxial pieces in their mind and then claim that every piece produces a torque because the large intensity gradient near the boundary of the piece results in azimuthal components to the momentum density.

I think this is not correct. An intensity gradient near a wall of a beam results in the azimuthal components only in the case of a real beam satisfying the Maxwell equations. There are no azimuthal components in a piece of a wave that is simply cut off from a whole wave. Such a piece cannot be considered at all because it does not satisfy the Maxwell equations. 


\section{Absorbing of the spin flux density}

Now we consider an addition to the tensor density $T_{\wedge}^{i k}$ of Section 2 . The tensor density $T_{\wedge}^{i k}$ of this section is due to absorbing of the spin angular momentum flux density (15) by the central region of the target. The field of the central region of the beam is (18)

$$
\boldsymbol{E}=\exp (\mathrm{i} z-\mathrm{i} t)(\boldsymbol{x}+\mathrm{i} \boldsymbol{y}) E_{0}(0), \quad \boldsymbol{B}=-\mathrm{i} \boldsymbol{E}, \quad x^{2}+y^{2}<R_{0}^{2} .
$$

The point $R_{0}$ (see Figure 1) satisfies the equation

$$
\int_{0}^{R_{0}} E_{0}^{2}(0) 2 \pi r \mathrm{~d} r=1, \quad E_{0}^{2}(0)=1 /\left(\pi R_{0}^{2}\right) .
$$

The electromagnetic field (40), according to (15), gives the component of the spin tensor

$$
\Upsilon^{x y z}(0)=-\Re\left(\bar{A}^{x} \partial^{z} A^{y}-\bar{A}^{y} \partial^{z} A^{x}+\bar{\Pi}^{x} \partial^{z} \Pi^{y}-\bar{\Pi}^{y} \partial^{z} \Pi^{x}\right) / 4=E_{0}^{2}(0) .
$$

Here $A^{x}=-\int E^{x} \mathrm{~d} t, A^{y}=-\int E^{y} \mathrm{~d} t, \Pi^{x}=\int B^{x} \mathrm{~d} t, \Pi^{y}=\int B^{y} \mathrm{~d} t$, the over lines mark complex conjugate complex numbers, and $\partial^{z}=-\partial_{z}$. Now we obtain the cylindrical components of the spin tensor density by the formula $\Upsilon_{\wedge}^{i j k}=\partial_{a}^{i} \partial_{b}^{j} \partial_{c}^{k} \Upsilon^{a b c}\left(g_{\wedge}\right)^{1 / 2}$ where $\partial_{a}^{i}$ are the matrix elements (27). For example,

$$
\Upsilon_{\wedge}^{r \phi z}(0)=\partial_{x}^{r} \partial_{y}^{\phi} \Upsilon^{x y z}(0)\left(g_{\wedge}\right)^{1 / 2}+\partial_{y}^{r} \partial_{x}^{\phi} \Upsilon^{y x z}(0)\left(g_{\wedge}\right)^{1 / 2}=E_{0}^{2}(0) .
$$

As is well known, the local conservation law $\nabla_{k} T_{\wedge}^{i k}=0$ is accompanied by the angular momentum conservation law (see, e.g. [31], p. 64)

$$
\nabla_{k} Y_{\wedge}^{i j k}-2 T_{\wedge}^{[i j]}=0 .
$$

In our case

$$
2 T_{3}{ }_{\wedge}^{[r \phi]}=\partial_{z} Y_{\wedge}^{r \phi z} \quad \text { if } \quad r<R_{0},
$$

where $T_{3 \wedge}^{[r \phi]}$ is the antisymmetric part of the 3-dimensional stress tensor density in the material of the absorbing plane. Using Equation (45), we arrive at an antisymmetric stress tensor, which characterizes a medium absorbing angular momentum flux. Integrating Equation (45) over $z$ yields for $r<R_{0}$

$$
\int_{0}^{\infty} 2 T_{3}^{[r \phi]} \mathrm{d} z=\int_{0}^{\infty} \partial_{z} Y_{\wedge}^{r \phi z} \mathrm{~d} z, \quad \text { i.e. } \quad 2 T_{\wedge}^{[r \phi]}=-Y_{\wedge}^{r \phi z}(0),
$$

where $T_{\wedge}^{r \phi}=\int_{0}^{\infty} T_{3 \wedge}^{r \phi} \mathrm{d} z$ stands for 2-tensor density as well as in Section 2. Thus, for $r<R_{0}$

$$
\begin{aligned}
& T_{\wedge}^{\phi r}=-T_{\wedge}^{r \phi}=T^{\hat{\phi} r}=-T^{r \hat{\phi}}=Y_{\wedge}^{r \phi z}(0) / 2=Y^{r \hat{\phi} z}(0) / 2=E_{0}^{2}(0) / 2=1 /\left(2 \pi R_{0}^{2}\right), \\
& T_{\wedge}^{r r}=T_{\wedge}^{\phi \phi}=0 .
\end{aligned}
$$

This means that the edge of a disk of radius $r$, which is cut off from the plane, i.e. a circle of radius $r$, acts on the remainder of the plane with a torque. The force acting on an element $\mathrm{d} l$ of the edge is $\mathrm{d} F^{\hat{\phi}}=T^{\hat{\phi} r} \mathrm{~d} l$, and the torque corresponding to the element is 
$\mathrm{d} \tau=r T^{\hat{\phi} r} \mathrm{~d} l$. Integrating yields

$$
\tau_{\text {spin }}=\int_{0}^{2 \pi r} r T^{\hat{\phi} r} \mathrm{~d} l=r^{2} / R_{0}^{2}, \quad r<R_{0} .
$$

Thus, material of the central region transmits the received torque to the periphery by inner tangential stress of (45), and the torque increases with $r$.

The result (48) can be obtained in another way. By definition of $\Upsilon_{\wedge}^{r \phi z}, \mathrm{~d} \tau^{r \phi}=\Upsilon_{\wedge}^{r \phi z} \mathrm{~d} a_{z}^{\wedge}$, i.e. $\mathrm{d} \tau=Y^{r \hat{\phi} z}(0) 2 \pi r \mathrm{~d} r$. So a disk of radius $r$, which is cut off from the plane, experiences the torque

$$
\tau_{\text {spin }}=\int_{0}^{r} E_{0}^{2}(0) 2 \pi r \mathrm{~d} r=E_{0}^{2}(0) \pi r^{2}=r^{2} / R_{0}^{2}, \quad r<R_{0} .
$$

At the edge of the beam, $r=R_{0}$, Equation (44) is changed to

$$
T^{[i j]}=0, \quad r>R_{0} .
$$

So, for $r>R_{0}, T^{\phi r}=T^{r \phi}$, i.e. the stress tensor is symmetric. But $T^{\phi r}(r)$ must be continuous. Thus, $T^{r \phi}$ changes its sign at $r=R_{0}: T^{r \phi} \rightarrow-T^{r \phi}$.

$$
T_{\wedge}^{\phi r}=T_{\wedge}^{r \phi}=1 / 2 \pi r^{2}, \quad r>R_{0},
$$

as well as in (35). A plot of $T^{\hat{\phi} r}(r)$ and $T^{r \hat{\phi}}(r)$ is given in Figure 3. So,

$$
\tau_{\text {spin }}=\int_{l=0}^{l=2 \pi r} r T^{\hat{\phi} r} \mathrm{~d} l=1 \quad \text { for } \quad r>R_{0} .
$$

We denote the torque (36), (37) $\tau_{\text {orbit }}$ because the torque arises from the first term of (12), which is an orbital angular momentum, and we denote the torque (49), (52) $\tau_{\text {spin }}$ because the torque arises from the second term of (12), which is the spin angular momentum. The total torque is

$$
\begin{array}{ll}
\tau=2 \pi C(r)+r^{2} / R_{0}^{2} & \text { for } \quad r<R_{0}, \\
\tau=2 & \text { for } \quad r>R_{0},
\end{array}
$$

according to (17).

\section{Vain attempts to find a spin inside the orbital angular momentum}

According to the paradigm (11), physicists try to decompose the moment of momentum (2), (11) into spin and orbital parts [32]. In particular, it has been proven [33, 34] that the moment of the Poynting vector of a circularly polarized beam with a plane phase front (18), i.e. the orbital angular momentum of the circulating flow of the beam (see Figure 1), i.e. integral over the wall region of the beam, equals spin, i.e. integral of the canonical component $Y_{c}^{x y 0}(6)$ (which coincides with our component $Y^{x y 0}(15)$ ) over the body of the 

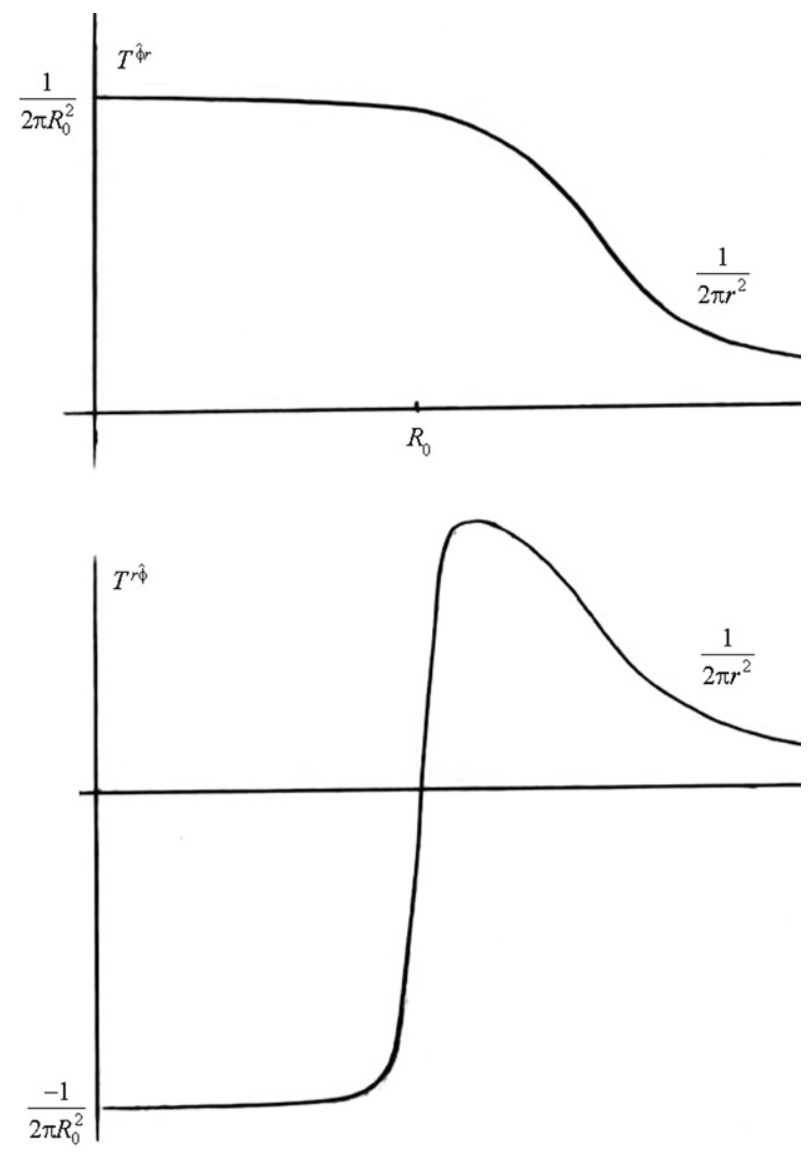

Figure 3. The components of the stress tensor arisen from the spin angular momentum.

beam (the Humblet transformaton):

$$
\int \boldsymbol{r} \times(\boldsymbol{E} \times \boldsymbol{B}) \mathrm{d} V=\int \boldsymbol{E} \times \boldsymbol{A} \mathrm{d} V .
$$

However, in our opinion, Equation (54) does not prove that orbital angular momentum is spin.

The proof of the Humblet transformation (54) uses $\boldsymbol{B}=\nabla \times \boldsymbol{A}$. The integrand of (11) is written as

$$
\boldsymbol{r} \times(\boldsymbol{E} \times \boldsymbol{B})=\boldsymbol{r} \times[\boldsymbol{E} \times(\nabla \times \boldsymbol{A})]=\boldsymbol{r} \times\left(E^{i} \nabla A_{i}\right)-\boldsymbol{r} \times[(\boldsymbol{E} \cdot \nabla) \boldsymbol{A}] .
$$

The first term in the right-hand side is zero for the beam (18), (19). Really, the Lorentz gauge vector potential corresponding to (18) is (see e.g. [35])

$$
\boldsymbol{A}=\exp (\mathrm{i} z-\mathrm{i} t)(-\mathrm{i} \boldsymbol{x}+\boldsymbol{y}) E_{0} .
$$




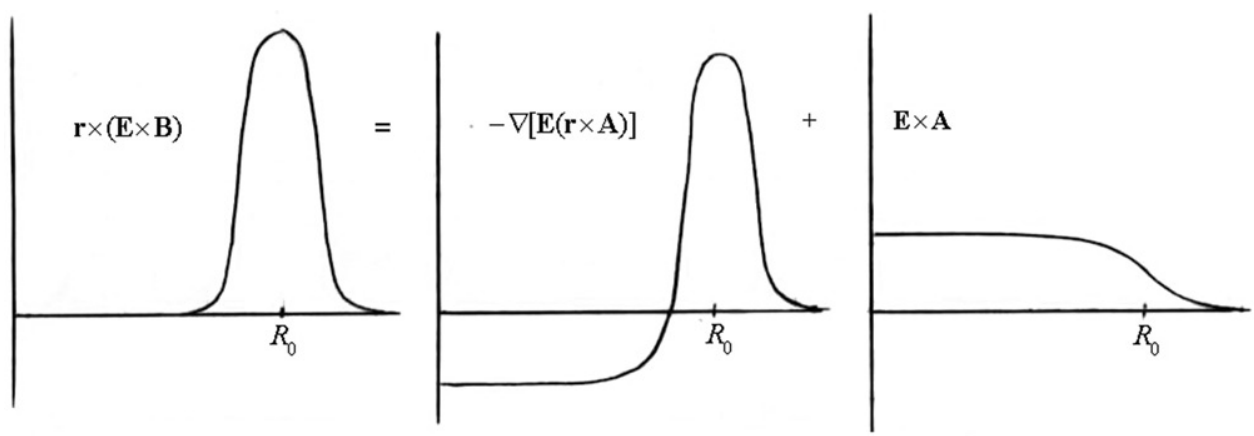

Figure 4. The term $\boldsymbol{E} \times \boldsymbol{A}$ is subtracted from $\boldsymbol{r} \times \boldsymbol{E} \times \boldsymbol{B}$ and then is added to $\boldsymbol{r} \times \boldsymbol{E} \times \boldsymbol{B}$.

So,

$$
\Re\left\{x \bar{E}^{x} \partial_{y} A_{x}+x \bar{E}^{y} \partial_{y} A_{y}-y \bar{E}^{x} \partial_{x} A_{x}-y \bar{E}^{y} \partial_{x} A_{y}\right) / 2=\Re\left\{\mathrm{i} \bar{E}_{0}\left(-x \partial_{y}+y \partial_{x}\right) E_{0}\right]=0 .
$$

The second term in the right-hand side of (55), according to the standard procedure, is augmented by the zero, $0=-\boldsymbol{E} \times \boldsymbol{A}+\boldsymbol{E} \times \boldsymbol{A}$. Then the second term is written as

$$
-\boldsymbol{r} \times[(\boldsymbol{E} \cdot \nabla) \boldsymbol{A}]=-\boldsymbol{r} \times[(\boldsymbol{E} \cdot \nabla) \boldsymbol{A}]-\boldsymbol{E} \times \boldsymbol{A}+\boldsymbol{E} \times \boldsymbol{A}=-\nabla[\boldsymbol{E}(\boldsymbol{r} \times \boldsymbol{A})]+\boldsymbol{E} \times \boldsymbol{A} .
$$

I duplicate here the equality $\nabla[\boldsymbol{E}(\boldsymbol{r} \times \boldsymbol{A})]=\boldsymbol{r} \times[(\boldsymbol{E} \cdot \nabla) \boldsymbol{A}]+\boldsymbol{E} \times \boldsymbol{A}$ in the index form

$$
\partial_{i}\left(E^{i} r^{[j} A^{k]}\right)=E^{i} r^{[j} \partial_{i} A^{k]}+E^{i} \delta_{i}^{[j} A^{k]} .
$$

So, the integrand of (11) takes the form

$$
\boldsymbol{r} \times(\boldsymbol{E} \times \boldsymbol{B})=-\nabla[\boldsymbol{E}(\boldsymbol{r} \times \boldsymbol{A})]+\boldsymbol{E} \times \boldsymbol{A} .
$$

Equation (60) is depicted in Figure 4 (compare with Figure 1). When integrating, the first term on the right gives zero and we arrive at the famous Equation (54). However, the equation only expresses a change from integrating over the wall region of the beam to integrating over the bulk. This change does not prove that the torque acts on the central region of the target, and that the moment of the Poynting vector is spin. This change of the integrating region proves nothing. For example, consider an analogous integral $\int \boldsymbol{r} \times \boldsymbol{j} \mathrm{d} V=\int \boldsymbol{r} \times(\nabla \times \boldsymbol{H}) \mathrm{d} V$ over the surface of a long solenoid where $\boldsymbol{j}$ is an electric current density of the solenoid. We have

$$
\begin{aligned}
\int \boldsymbol{r} \times(\nabla \times \boldsymbol{H}) \mathrm{d} V & =\int\left(r^{i} \partial_{k} H_{i}-r^{i} \partial_{i} H_{k}\right) \mathrm{d} V \\
& =\int\left[\partial_{k}\left(r^{i} H_{i}\right)-H_{k}-\partial_{i}\left(r^{i} H_{k}\right)+\partial_{i} r^{i} H_{k}\right] \mathrm{d} V \\
& =\int 2 \boldsymbol{H} \mathrm{d} V .
\end{aligned}
$$

This equality between the moment of an electric current and the integral of $\boldsymbol{H}$ over the solenoid volume proves nothing. 
Another transformation of the angular momentum (11) is offered by Stewart $[25,26]$. He uses the electric vector potential $\Pi$ (see Section 1) instead of the magnetic vector potential $\boldsymbol{A}$, and he obtains

$$
\int \boldsymbol{r} \times(\boldsymbol{E} \times \boldsymbol{B}) \mathrm{d} V=\int \Pi \times \boldsymbol{B} \mathrm{d} V
$$

instead of (54) for the beam (18). But this result can be easily obtained if one rewrites Equation (55) as

$$
\begin{aligned}
\boldsymbol{r} \times(\boldsymbol{E} \times \boldsymbol{B}) & =-\boldsymbol{r} \times(\boldsymbol{B} \times \boldsymbol{E})=-\boldsymbol{r} \times[\boldsymbol{B} \times(\nabla \times \Pi)] \\
& =-\boldsymbol{r} \times\left(B^{i} \nabla \Pi_{i}\right)+\boldsymbol{r} \times[(\boldsymbol{B} \cdot \nabla) \Pi],
\end{aligned}
$$

i.e. changes $\boldsymbol{E} \rightarrow \boldsymbol{B}, \boldsymbol{A} \rightarrow \Pi$.

It must be noted that $\Pi \times \boldsymbol{B}$ is a magnetic alternative to the component $Y_{c}^{x y 0}$ of the invalid canonical spin tensor (5) and was presented in [36]. For the beam (18), $\boldsymbol{E} \times \boldsymbol{A}=\Pi \times \boldsymbol{B}$.

\section{Conclusions}

This paper conveys new physics. We review existing works concerning electrodynamics spin and indicate that existing theory is insufficient to solve spin problems because the spin tensor of modern electrodynamics is zero. Then we show how to resolve the difficulty by introducing a true electrodynamics spin tensor. Our spin tensor doubles a predicted angular momentum of a circularly polarized light beam without an azimuth phase structure. The tensor is needed, in particular, for understanding of essential characteristic features of a rotating dipole radiation [16].

\section{Acknowledgements}

I am deeply grateful to Professor Robert H. Romer for valiant publishing of my question [37] (submitted on 7 October 1999) and to Professor Timo Nieminen for valuable discussions (Newsgroups: sci.physics.electromag).

\section{References}

[1] Heitler, W. The Quantum Theory of Radiation; Clarendon: Oxford, 1954, p 401.

[2] Rohrlich, F. Classical Charged Particles; Addison-Wesley: Reading, MA, 1965.

[3] Khrapko, R.I. Moment of the Poynting Vector is not Spin, V. 2, 2006, poynting_vector_ khrapko.pdf, www.sciprint.org (accessed April 17, 2004).

[4] Zambrini, R.; Barnett, S.M. J. Mod. Opt. 2005, 52, 1045-1052.

[5] Belinfante, F.J. Physica 1939, 6, 887.

[6] Rosenfeld, L. Mem. Acad. R. Sci. Belg. 1940, 8 (6), 1-30.

[7] Jackson, J.D. Classical Electrodynamics; Wiley: New York, 1999, p 350.

[8] Soper, D.E. Classical Field Theory; Wiley: New York, 1976.

[9] Loudon, R. Phys. Rev. A 2003, 68, 013806.

[10] Bishop, A.I.; Nieminen, T.A.; Heckenberg, N.R.; et al. Phys. Rev. Lett. 2004, 92, 198104. 
[11] Khrapko, R.I. True Energy-momentum Tensors are Unique. Electrodynamics Spin Tensor is not Zero. http://arXiv.org/abs/physics/0102084.

[12] Khrapko, R.I. Measurement Techn. 2003, 46 (4), 317.

[13] Khrapko, R.I. Gravitation \& Cosmology 2004, 10 (1-2), 91.

[14] Khrapko, R.I. Absorption of a Circularly Polarized Beam in a Dielectric, etc., 2006, www.sciprint.org (accessed April 6, 2007).

[15] Khrapko, R.I. Inevitability of the Electrodynamics' Spin Tensor, 2007, Inevitability_ spin_khrapko_mar07. www.sciprint.org (accessed March 20, 2007).

[16] Khrapko, R.I. Radiation of Spin by a Rotator, mp_arc@mail.ma.utexas.edu REQUEST: send papers NUMBER: 03-315 (accessed June 28, 2003).

[17] Simpson, N.B.; Dholakia, K.; Allen, L.; et al. Opt. Lett. 1997, 22, 52.

[18] Parkin, S.; Knoner, G.; Nieminen, T.A.; et al. Opt. Express 2006, 14, 6963.

[19] Garces-Chavez, V.; McGloin, D.; Padgett, M.J.; Dultz, W.; Schmitzer, H.; Dholakia, K. Phys. Rev. Lett. 2003, 91, 093602.

[20] O'Neil, A.T.; et al. Phys. Rev. Lett. 2002, 88, 053601.

[21] Beth, R.A. Phys. Rev. 1936, 50, 115.

[22] Friese, M.E.J.; Enger, J.; Heckenberg, N.R.; et al. Phys. Rev. A 1996, 54, 1593.

[23] Ohanian, H.C. Am. J. Phys. 1986, 54, 500.

[24] Simmonds, J.W.; Guttmann, M.J.; States, Waves and Photons; Addison-Wesley: Reading, MA, 1970.

[25] Stewart, A.M. Eur. J. Phys. 2005, 26, 635.

[26] Stewart, A.M. J. Mod. Opt. 2005, 52, 408.

[27] Kogelnik, H.; Li, T. Appl. Opt. 1966, 5, 1550.

[28] Schouten, J.A.; Tensor Analysis for Physicists; Clarendon: Oxford, 1951.

[29] Feynman, R.P.; Leighton, R.B.; Sands, M. The Feynman Lectures on Physics; Addison-Wesley: London, 1965, Vol. 3, pp 17-10.

[30] Allen, L.; Padgett, M.J. Am. J. Phys. 2002, 70, 567.

[31] Hehl, F.W. Rep. Math. Phys. 1976, 9 (1), 55.

[32] Crichton, J.H.; Marston, P.L. Electron. J. Diff. Equations Conf. 2000, 4, 37-50.

[33] Humblet, J.; Physica 1943, 10, 585.

[34] Crichton, J.; et al. Gen. Relat. Grav. 1990, 22, 61.

[35] Allen, L.; Padgett, M.J.; Babiker, M. Progress in Optics XXXIX; Elsevier: Amsterdam, 1999, p 298.

[36] Khrapko, R.I. Electrodynamics' Spin, 2005, electrodynamics_spin_khrapko. http://www. sciprint.org (accessed April 5, 2007).

[37] Khrapko, R.I. Am. J. Phys. 2001, 69, 405. 